Artigo Original

\title{
Comparação de indicadores físicos e fisiológicos entre atletas profissionais de futsal e futebol
}

\author{
Renan Felipe Hartmann Nunes \\ Flávia Angélica Martins Almeida \\ Bruno Vinícius Santos \\ Fabrícia Daniela Martins Almeida \\ Gustavo Nogas \\ Hassan Mohamed Elsangedy \\ Kleverton Krinski \\ Sergio Gregório da Silva

\section{Departamento de Educação Física, Centro de Pesquisa em Exercício e Esporte da Universidade Federal do Paraná, Curitiba, PR, Brasil}

\begin{abstract}
Resumo: Muitos profissionais do esporte periodizam o treinamento do futebol e do futsal de forma semelhante. Todavia, pouco se conhece das respostas físicas e fisiológicas dos atletas de futsal. Esse estudo comparou perfil antropométrico, capacidade aeróbia e produção de potência entre atletas profissionais de futsal e futebol. Onze jogadores de futsal (idade $24,1 \pm 2,4$ anos) e 21 jogadores de futebol (idade 22,6 $\pm 3,6$ anos) do sexo masculino submeteram-se a duas sessões experimentais: (I) avaliação antropométrica, teste de esforço máximo em esteira e (II) teste de velocidade de 30 metros (capacidade de sprint repetido, CSR). Empregou-se teste $t$ para amostras independentes, permitindo verificar possíveis diferenças entre as modalidades $(p<0,05)$. Comparados aos atletas de futebol, os jogadores de futsal apresentaram, respectivamente, maiores valores de $\mathrm{FC}_{\mathrm{LV}}(177,2 \pm 10$ e $167,1 \pm 10,8 \mathrm{bpm}) ; \mathrm{VO}_{2 \max }, \mathrm{VO}_{2\llcorner\mathrm{~V}} \mathrm{e}$ $\% \mathrm{VO}_{2 \max }\left(62,5 \pm 4,3\right.$ e $52,1 \pm 4,6 ; 58,7 \pm 5,6$ e $43,1 \pm 4,6 ; 93,9 \pm 5,3$ e $\left.76 \pm 8,4 \mathrm{ml} \cdot \mathrm{kg}^{-1} \cdot \mathrm{min}^{-1} 1\right)$. Esses resultados demonstram a necessidade de métodos específicos para treinamento do futsal e futebol.
\end{abstract}

Palavras-chave: Futsal. Futebol. Consumo máximo de oxigênio. Potência muscular.

\section{Comparison of physical and physiological indicators between professional futsal and soccer athletes}

\begin{abstract}
Many professional sports training periodize soccer and futsal similarly. However, little is known about physical and physiological responses of futsal players. This study compared anthropometric, aerobic capacity and power production among professional indoor and outdoor soccer players. Eleven futsal players

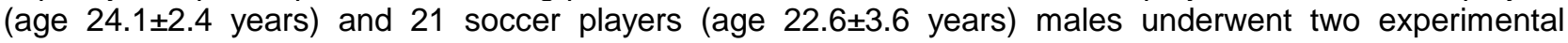
sessions: (i) anthropometric measurements, maximal effort test treadmill and (II) test speed of 30 meters (repeated sprint ability, CSA). It was applied t-test for independent samples to verify possible differences between the modalities $(p>0,05)$. The futsal players showed higher values of $\mathrm{HR}_{\mathrm{VT}}(177,2 \pm 10$ e $67,1 \pm 10,8$ bpm) $\mathrm{VO}_{2 \max } ; \mathrm{VO}_{2 \mathrm{VT} ;} \% \mathrm{VO}_{2 \max }\left(62,5 \pm 4,3\right.$ e $52,1 \pm 4,6 ; 58,7 \pm 5,6$ e $43,1 \pm 4,6 ; 76 \pm 8,4$ e $\left.93,9 \pm 5,3 \mathrm{ml} \cdot \mathrm{kg}^{-1} \cdot \mathrm{min}^{-1}\right)$ compared with soccer players, respectively. These results demonstrate the need for specific methods of training for outdoor and indoor soccer.
\end{abstract}

Keywords: Futsal. Soccer. Maximal oxygen uptake. Muscle power.

\section{Introdução}

Considerado uma versão indoor do futebol, o futsal é um esporte de característica de elevada intensidade, intermitente e com atividades acíclicas. Esta modalidade, praticada no mundo todo (12 milhões de jogadores em mais de 100 países) enfatiza a velocidade de corrida e resistência física, e requer níveis substanciais de força para chutes, arranques, mudanças rápidas de direção e capacidade de sprints repetidos (CSR) durante as ações dos jogos (BARBEROALVAREZ et al., 2008; GOROSTIAGA et al., 2009). Tais características e demandas podem ser explicadas pelo fato do futsal apresentar um número ilimitado de substituições, por isso, os níveis de intensidade durante a partida são extremamente elevados, sem diminuição de desempenho durante 0 jogo (BARBEROALVAREZ; GIMENEZ; CORONA; MANONELLES, 2002).

Como no futsal, o futebol também é um esporte com características intermitentes, de intensidade extenuante com ênfase nos componentes de força, velocidade e resistência (GOROSTIAGA et al., 2009). Diferente do futsal, 
- futebol apresenta maiores dimensões e prolongado tempo de jogo, maior quantidade de atletas e com poucas paradas do cronômetro, além disso, apresenta um número limitado de substituições. Devido ao longo período de uma partida de futebol, grande parte da sua liberação de energia provém do metabolismo aeróbio (BANGSBO, 1994), além disso, durante um jogo, os atletas percorrem em média $10 \mathrm{~km}$ (BANGSBO, 1991; HELGERUD, 2001) em uma intensidade próxima ao limiar anaeróbio ou 80$90 \%$ da freqüência cardíaca máxima (BANGSBO, 1994; HELGERUD, 2001; REILLY,1984).

Alguns estudos foram conduzidos comparando o futsal com o futebol de campo devido à semelhança das características físicas, técnicas ou táticas entre estas modalidades (GOROSTIAGA et al., 2009; LEAL JUNIOR et al., 2006). Contudo, Barbero-Alvarez (2008) demonstrou que o percentual da distância total percorrida em velocidade máxima e o percentual da frequência cardíaca registrada durante o jogo de futsal foram superiores quando comparadas com futebol, e verificou-se que a frequência cardíaca durante a partida de futsal permaneceu acima de $85 \%$ da frequência cárdica máxima por mais de $80 \%$ do tempo do jogo em quadra, resultados que sugerem o futsal como um dos esportes com maiores exigências físicas.

Como consequência das diferentes características entre o futebol e futsal, tem-se argumentado que possa haver diferenças físicas entre essas modalidades. Poucos estudos têm verificado as características antropométricas (GOROSTIAGA et al., 2009; BARBEROALVAREZ et al., 2008; CASTAGNA et al., 2009; AVELAR, et al., 2008), fisiológicas (GOROSTIAGA et al., 2009; CASTAGNA et al., 2009; BARBERO-ALVAREZ et al., 2009; LEAL JUNIOR et al., 2006; LIMA; SILVA; SOUZA, 2005) e produção de potência (GOROSTIAGA et al., 2009) em atletas de futsal, pois em sua maioria os estudos tem apenas verificado essas variáveis físicas em atletas de futebol (STOLEN et al., 2005; ARNASON et al., 2004; BOSCO et al.,1996; CASAJUS 2001; RIENZI et al., 2000). Como são modalidades similares em seus gestos esportivos, porém com grande discrepância em relação às dimensões físicas em que são praticadas (tamanho da quadra), indaga-se quais seriam também as semelhanças e diferenças encontradas nos parâmetros antropométricos, capacidade aeróbia e produção de potência de seus praticantes.

Nesse sentido, informações sobre as características dos atletas e os efeitos do treinamento sistematizado do futsal e futebol têm sido disponibilizados na literatura. Todavia, pouco se conhece sobre as respostas físicas e fisiológicas de atletas de futsal, e se existe diferença entre as modalidades, pois devido a semelhança motora entre as modalidades muitos profissionais periodizam treinamento com mesmo volume e intensidade, sendo que as características de jogo são diferentes.

Tais informações parecem ser relevantes para a escolha das estratégias de preparação física a serem aplicadas. Dessa forma, o presente estudo teve como objetivo descrever e comparar o perfil antropométrico, a capacidade aeróbia e produção de potência de atletas profissionais de futsal e futebol de campo.

\section{Sujeitos}

O presente estudo foi composto por 32 homens atletas, sendo 11 atletas de futsal de uma equipe que disputam o campeonato paranaense e liga nacional e 21 atletas de futebol que disputa 0 campeonato paranaense da $1^{\circ}$ divisão. Todos apresentavam ao menos 5 anos de experiência em competições e realizavam treinamentos com frequência mínima de cinco vezes por semana e quatro horas diárias.

Antes do início do estudo, os atletas foram submetidos a uma avaliação médica inicial para identificar a existência de alguma desordem física a qual poderia limitar sua participação no estudo.

Todos os sujeitos foram informados sobre os procedimentos utilizados, possíveis benefícios e riscos atrelados à execução do estudo, condicionando posteriormente a sua participação de modo voluntário através da assinatura do termo de consentimento livre e esclarecido conforme as diretrizes propostas na Resolução 196/96 do Conselho Nacional de Saúde sobre pesquisas envolvendo seres humanos.

\section{Delineamento Experimental}

Os participantes foram submetidos a duas sessões experimentais em dias distintos com um intervalo mínimo de 48 horas. Na primeira sessão, laboratorial, os sujeitos assinaram o termo de consentimento livre e esclarecido seguido da avaliação antropométrica, familiarização com os equipamentos e instruções referentes à sessão 
experimental. Em seguida, foi conduzido um teste incremental até exaustão volitiva em esteira para a obtenção dos parâmetros fisiológicos máximos e relativos ao limiar ventilatório. Todos os participantes foram instruídos a não realizar atividade física vigorosa nas 24 horas anteriores aos testes, bem como não ingerirem qualquer tipo alimento e bebidas cafeinadas por um período de duas horas antecedentes ao seu início. A média de temperatura ao longo dos testes foi de $24 \pm 0.5$ ${ }^{\circ} \mathrm{C}$.

Na segunda sessão, após familiarização com os equipamentos e procedimentos, foi conduzido um teste de velocidade de 30 metros (capacidade de sprint repetido, CSR), com o intuito de verificar os valores de produção de potência e índice de fadiga.

\section{Teste incremental até a exaustão em esteira}

Os atletas realizaram um aquecimento em esteira (marca InBramed ${ }^{\circledR}$, modelo Super ATL, POA, Brasil) de 2 minutos a uma velocidade de $6,0 \mathrm{~km} \cdot \mathrm{h}^{-1}$ e $0 \%$ de inclinação. Posteriormente, um teste de esteira incremental foi conduzido em uma velocidade de $8,0 \mathrm{~km} \cdot \mathrm{h}^{-1}$ e $0 \%$ de inclinação, mantendo-se por 6 minutos. Após isso, a velocidade foi aumentada $1,0 \mathrm{~km} \cdot \mathrm{h}^{-1}(0 \%$ de inclinação) a cada minuto até a exaustão volitiva (BARBERO-ALVAREZ et al., 2009). Os participantes foram encorajados verbalmente a se manter em exercício pelo maior tempo possível. A escolha desse protocolo de teste incremental deve-se ao seu emprego em prévios estudos envolvendo uma população similar (BARBEROALVAREZ et al., 2009; CASTAGNA et al., 2009). Durante toda a realização do teste, parâmetros fisiológicos máximos e referentes ao limiar ventilatório foram obtidos.

\section{Teste de velocidade de 30 metros (Capacidade de Sprint Repetido CSR)}

O teste foi constituído de dez sprints máximos de 30 metros com intervalo de recuperação de 10 segundos entre cada sprint, realizado em linha reta sem mudança de direção, assim, os valores de potência mínima, máxima e média foram obtidos. O índice de fadiga foi realizado através de um cálculo que demonstra um percentual de queda entre os 10 tiros, sendo que, esse índice é uma variável importante, pois nos dá uma estimativa da resistência de trabalho anaeróbio e da possibilidade de sustentar sprints repetidos, fator de grande importância no futsal e futebol.
Nesse sentido, quanto maior for 0 índice de fadiga, maior será a queda entre os sprints, o que significa que o atleta apresenta pouca resistência de velocidade.

\section{Instrumentos e Procedimentos}

As variáveis antropométricas massa corporal (MC, em kg.; balança marca Toledo, modelo 2096), e estatura (EST, em cm; estadiômetro marca Sanny, modelo Standard) foram obtidas conforme os procedimentos propostos por Gordon et al., (1988). A densidade corporal (em $\mathrm{g} / \mathrm{cm}^{3}$ ) foi determinada através da utilização do método de espessura de dobras cutâneas, de acordo com a equação proposta por Jackson e Pollock (1985), mediante a utilização de um compasso da marca Lange ${ }^{\circledR}$ (pressão constante de $10 \mathrm{~g} / \mathrm{mm}^{2}$ ). Posteriormente, o percentual de gordura corporal (\% gordura) foi obtido mediante utilização da equação de Siri (1961). Buscando evitar variações inter-avaliadores, todas as medidas foram obtidas por um único avaliador previamente treinado.

$\mathrm{O} \mathrm{VO}_{2}$ (em $\mathrm{mL} \cdot \mathrm{kg}^{-1} \cdot \mathrm{min}^{-1}$ ) foi mensurado continuamente durante a realização do testes de esteira, através da utilização de um sistema de espirometria computadorizado de circuito aberto (marca Parvomedics ${ }^{\circledR}$, modelo TrueMax 2400, Salt Lake City, Estados Unidos). Anteriormente a realização de cada avaliação, o sistema foi calibrado tanto para $\mathrm{O}_{2}$ e $\mathrm{CO}_{2}$, através da utilização de uma concentração gasosa padronizada de $\mathrm{O}_{2}$ e $\mathrm{CO}_{2}$, como também para a ventilação, mediante o uso de uma seringa de 3 litros (marca Hans Rudolph ${ }^{\circledR}$, modelo 5530, Kansas City, Estados Unidos)

$\mathrm{O} \dot{V} \mathrm{O}_{2 \mathrm{Max}}$ e o consumo de oxigênio no limiar ventilatório $\left(\mathrm{VO}_{2 \mathrm{LV}}\right)$ foram determinados como $\mathrm{o}$ maior $\mathrm{VO}_{2}$ médio (intervalo de 1 minuto) verificado no último estágio completo do teste de esteira incremental e no limiar ventilatório, respectivamente (CAIOZZO et al., 1982). A determinação do $\mathrm{VO}_{2 \max }$ baseou-se em dois dos seguintes critérios: (a) platô do $\mathrm{VO}_{2}$, indicado por uma diferença de $<2,1 \mathrm{~mL} \cdot \mathrm{Kg}^{-1} \cdot \mathrm{min}^{-1}$ entre os dois últimos estágios do teste; (b) taxa de troca respiratória (RER) > 1,10; e (c) frequência cardíaca dentro de $\pm 10 \mathrm{bpm}$ para cada sujeito através do valor máximo predito pela idade (CAIOZZO et al., 1982). O limiar ventilatório (LV) foi calculado individualmente através do método de equivalente ventilatório, considerada a intensidade na qual há o primeiro aumento súbito 
no equivalente ventilatório de oxigênio $\left(\mathrm{VE} / \mathrm{VO}_{2}\right)$ sem alterações no equivalente ventilatório de dióxido de carbono $\left(\mathrm{VE} / \mathrm{VCO}_{2}\right)$. Uma avaliação a posteriori para determinar o LV foi conduzida por dois experientes avaliadores, sendo determinada como LV o primeiro ponto de quebra onde houve concordância nas identificações (AIOZZO et al., 1982).

A $F C_{\max }$ e frequência cardíaca no limiar ventilatório $\left(\mathrm{FC}_{\mathrm{LV}}\right)$ foram determinadas como a maior FC média (intervalo de 1 minuto) verificada no último estágio completo do teste de esteira incremental e no limiar ventilatório, respectivamente.

A velocidade de deslocamento de 30 metros foi mensurada continuamente durante a realização do teste através de um equipamento de gravação do tempo denominado de células fotoelétricas (marca Microgate ${ }^{\circledR}$, Polifemo, Itália). As células fotoelétricas foram posicionadas no início do sprint $(0 \mathrm{~m})$ e aos $30 \mathrm{~m}$, colocado $0,4 \mathrm{~m}$ acima do solo, com uma precisão de 0,001 s. Assim, para iniciar o teste o atleta permaneceu parado com um dos pés que lhe fosse mais confortável próximo ao $1^{\circ}$ par de célula fotoelétrica, e após a liberação do sistema pelo avaliador o atleta iniciou o teste no momento que
Ihe fosse conveniente. A partir do momento em que o atleta passou a perna entre o 1ำ par 0 sistema ativou o cronômetro do sistema de sensores fotoelétricos, assim, quando o mesmo ultrapassou pelo $2^{\circ}$ par, aos 30 metros, foi estabelecido a primeira medida de tempo. Através dos valores de cada sprint (cada velocidade de 10 m) a potência mínima, máxima e média, além, do índice de fadiga, foram obtidos, derivados das seguintes fórmulas.

$$
\text { Potência }=\text { Peso } \times \text { Distância }^{2} / \text { Tempo }^{3}
$$

O cálculo do Índice de fadiga foi determinado pela equação: IF = (PMax - PMin $) \times 100 /$ PMax.

\section{Análise Estatística}

Os resultados do presente estudo são apresentados como média e desvio-padrão. Antes dos testes paramétricos, a normalidade foi verificada através do teste de Shapiro-Wilk. Teste $T$ de amostras independentes foi usado para examinar possíveis diferenças entre as modalidades. $O$ valor de $p<0,05$ foi considerado como nível de significância estatística. Os procedimentos estatísticos do presente estudo foram realizados mediante a utilização do Statistical Package for the Social Sciences (SPSS, versão 13.0) for Windows.

\section{Resultados}

As características físicas dos atletas de futsal e futebol estão apresentados na tabela 1. Não foram encontradas diferenças significativas em nenhuma das variáveis.

Tabela1- Características físicas dos atletas de futsal e futebol

\begin{tabular}{ccc}
\hline & Atletas de futsal $(\mathbf{n = 1 1 )}$ & Atletas de futebol $(\mathbf{n}=\mathbf{2 1})$ \\
\hline Idade (anos) & $24,18 \pm 2,4$ & $22,67 \pm 3,6$ \\
Estatura $(\mathbf{c m})$ & $1,78 \pm 0,03$ & $1,77 \pm 0,07$ \\
Massa Corporal $(\mathbf{k g})$ & $78,6 \pm 10,3$ & $76 \pm 8,9$ \\
\% Gordura & $12,3 \pm 4,0$ & $11,3 \pm 3,2$ \\
\hline
\end{tabular}

Tabela 2- Características fisiológicas dos atletas de futsal e futebol

\begin{tabular}{|c|c|c|}
\hline & Atletas de futsal $(n=11)$ & Atletas de futebol $(n=21)$ \\
\hline $\mathrm{FC}_{\max }(\mathrm{bpm})$ & $183,1 \pm 8,7$ & $189 \pm 10,7$ \\
\hline $\mathrm{FC}_{\mathrm{Lv}}(\mathrm{bpm})$ & $177,2 \pm 10,1$ & $167,1 \pm 10,8^{*}$ \\
\hline $\begin{array}{c}\% \mathrm{FC}_{\operatorname{Max}}(\mathrm{bpm}) \\
\mathrm{VO}_{2 \max }\left(\mathrm{ml} \cdot \mathrm{kg}^{-1} \cdot \mathrm{min}^{-1}\right)\end{array}$ & $\begin{array}{l}96,7 \pm 2,3 \\
62,5 \pm 4,3\end{array}$ & $\begin{array}{l}88,4 \pm 2,4 \\
52,1 \pm 4,6^{*}\end{array}$ \\
\hline $\mathrm{VO}_{2 \mathrm{LV}}\left(\mathrm{ml} \cdot \mathrm{kg}^{-1} \cdot \mathrm{min}^{-1}\right)$ & $58,7 \pm 5,6$ & $43,1 \pm 4,6^{*}$ \\
\hline$\% \mathrm{VO}_{2 \max }\left(\mathrm{ml} \cdot \mathrm{kg}^{-1} \cdot \mathrm{min}^{-1}\right)$ & $93,9 \pm 5,3$ & $76 \pm 8,4^{*}$ \\
\hline
\end{tabular}

$\mathrm{FC}_{\mathrm{MAX}}=$ Frequência cardíaca máxima; $\mathrm{FC}_{\mathrm{LV}}=$ Frequência cardíaca no limiar ventilatório; $\% \mathrm{FC}_{\max }=$ Percentual da frequência cardíaca máxima; $\mathrm{VO}_{2 \max }=$ Consumo máximo de oxigênio; $\mathrm{VO}_{2 L V}=$ Consumo de oxigênio no limiar ventilatório; $\% \mathrm{VO}_{2 \max }=$ percentual do consumo máximo de oxigênio. 
Os valores de potência e índice de fadiga dos atletas de futsal e futebol estão apresentados na tabela 3. Não foram encontradas diferenças significativas entre as modalidades.

Tabela 3- Teste de sprint repetido de $30 \mathrm{mts}$ nos atletas de futsal e futebol

\begin{tabular}{ccc}
\hline & Atletas de futsal $(\mathbf{n}=\mathbf{1 1})$ & Atletas de futebol $(\mathbf{n}=\mathbf{2 1})$ \\
\hline PM (Watts) & $642,3 \pm 146,3$ & $637,5 \pm 73,6$ \\
PMin(Watts) & $504,1 \pm 133,3$ & $510,3 \pm 82,8$ \\
PMax(Watts) & $852,3 \pm 207,9$ & $844 \pm 95,1$ \\
IF (\%) & $7,2 \pm 2,7$ & $6,9 \pm 1,5$ \\
\hline
\end{tabular}

PM= Potência média; PMin= Potência mínima; PMax= Potência máxima; IF= Índice de fadiga.

\section{Discussão}

Esta pesquisa teve como propósito analisar e comparar as variáveis antropométricas, fisiológicas e produção de potência em atletas de elite de futsal e futebol.

Como resultado, os atletas de futsal apresentaram maiores valores de $\mathrm{FC}_{\mathrm{LV}} ; \mathrm{VO}_{2 \max }$; $\mathrm{VO}_{2 \mathrm{LV} ;} \% \mathrm{VO}_{2 \max }$, comparado aos atletas de futebol de campo (tabela 2). Esses resultados são diferentes aos achados de Leal Junior et al., (2006), que não encontraram diferença significativa nos valores de $\mathrm{VO}_{2 p i c o}$ durante um teste de esforço máximo entre atletas profissionais paulistas de futsal e futebol $(55,7 \mathrm{e}$ $54,8 \mathrm{ml} \cdot \mathrm{kg}^{-1} \cdot \mathrm{min}^{-1}$ ) respectivamente. Entretanto, apesar dos atletas de futsal permanecerem por maior tempo em condição anaeróbia, estes atingiram o limiar anaeróbio (2,97 L/min) em menor tempo em relação aos atletas de futebol (3,46 L/min).

Apesar da capacidade aeróbia não ser um elemento determinante do desempenho durante a partida de futsal, valores de $\mathrm{VO}_{2 \max }$ entre 50-55 $\mathrm{ml} \cdot \mathrm{kg}^{-1} \cdot \mathrm{min}^{-1}$ parecem ser aconselháveis para atletas profissionais dessa modalidade (CASTAGNA et al., 2009), principalmente para uma melhor recuperação de energia entre sprints repetidos. Os valores encontrados na literatura para essa variável, em atletas profissionais, são semelhantes aos obtidos no presente estudo (CASTAGNA et al., 2009; BARBERO-ALVAREZ et al., 2009; LIMA et al., 2005), mas superiores quando comparado com atletas semi profissionais (BARBERO-ALVAREZ et al., 2009).

Além da capacidade aeróbia como uma variável discriminante do futsal, este esporte, de característica intermitente e elevada demanda física necessita de valores que indiquem alta capacidade anaeróbia. Nesta pesquisa, os atletas de futsal apresentaram valores médios de $\% \mathrm{VO}_{2 \max }$ no LV e VO $\mathrm{LLV}(93,9 \% ; 58,7 \mathrm{ml} / \mathrm{kg} / \mathrm{min})$ superiores aos atletas espanhóis pesquisados por Barbero-Alvarez et al., (2009) e Castagna et al., (2009) $(70,5$ e $71 \% ; 44,4$ e $46 \mathrm{ml} / \mathrm{kg} / \mathrm{min})$, respectivamente. Isso demonstra elevada capacidade anaeróbia da amostra analisada, o que é um fator determinante na modalidade.

Já no futebol, as variáveis fisiológicas tem sido amplamente estudadas (STØLEN et al., 2005; BANGSBO, 1994; REILLY,1984), mas devido à grande diferença metodológica entre eles, tornase difícil a comparação. Porém, a literatura indica valores de referência de $\mathrm{VO}_{2 \max }$ entre $50-75 \mathrm{ml} \cdot \mathrm{kg}$ ${ }^{1} \cdot \mathrm{min}^{-1}$ nas diferentes posições de jogo (STØLEN et al., 2005; BANGSBO, 1994; EKBLOM,1986), valores superiores ao recomendado para o futsal.

Apresentar elevados valores de $\mathrm{VO}_{2 \max }$ no futebol parece ser mais interessante que no futsal, e essa importância se dá principalmente em posições que exigem maior volume de jogo, como em jogadores que atuam no meio de campo. Isso porque a exigência física imposta em ambos os esportes é diferente.

Algumas pesquisas tem utilizado a FC para identificar a intensidade de jogos em atletas de futsal (BARBERO-ALVAREZ et al., 2009) e futebol (BANGSBO, 1994; STØLEN et al., 2005). Os achados do nosso trabalho demonstraram que os atletas de futsal apresentaram maiores valores da $\mathrm{FC}_{\mathrm{LV}}(177,2$ e 167,1 bpm) quando comparados aos atletas de futebol, tabela 2. Barbero-Alvarez et al., (2008) encontraram valores médios da FC de $174 \mathrm{bpm}$, correspondendo a $90 \%$ da $\mathrm{FC}_{\operatorname{Max}}$ durante $72 \%$ do tempo. Os autores demonstraram também que a distância total percorrida (\%) em velocidade máxima durante a partida de futsal foi realizada acima de $85 \%$ da $\mathrm{FC}_{\text {Máx }}$ por mais de $80 \%$ do tempo em quadra. Além do mais, durante 
os curtos períodos de repouso, a FC raramente ficava abaixo de 150 bpm. Esses dados demonstram o grande contraste com a modalidade do futebol, tendo em vista que este, durante aproximadamente $90 \%$ do tempo de jogo, utiliza-se prioritariamente do metabolismo aeróbio (BANGSBO, 1994).

Além de maiores valores de $\mathrm{FC}_{\mathrm{LV}}$, os atletas de futsal apresentaram maiores valores do $\% \mathrm{VO}_{2 \max }(93,9 \%)$ comparado aos atletas de futebol (76\%), tabela 2. Uma explicação plausível para tais valores encontrados pode ser a diferente dinâmica de jogo entre os dois esportes. As maiores taxas de $\mathrm{FC}$ e $\% \mathrm{VO}_{2 \max }$ encontradas no futsal são, provavelmente, resultantes da elevada carga originada do metabolismo anaeróbio. Assim, atletas que apresentem um melhor condicionamento anaeróbio também apresentam uma facilitada remoção do ácido láctico pelas vias circulatórias, consequentemente diminuindo a segunda fonte de produção de $\mathrm{CO}_{2}\left(\mathrm{CO}_{2}\right.$ não metabólico), que ocorre acima do limiar anaeróbio e é resultante do tamponamento do lactato em níveis mais elevados de exercício (BEAVER et al., 1973).

Outro fator determinante para as modalidades em questão é a contração muscular explosiva, que tem sido identificada como um componente crucial no desempenho de realizar sprints (NEWMAN et al., 2004; ALEXANDER, 1989). Dowson et al., (1998) forneceu evidências de que a magnitude da força gerada durante a ação muscular dinâmica diz respeito à quantidade de velocidade que um atleta pode produzir durante uma performance de sprint. A capacidade de realizar sucessivos sprints na maior velocidade possível, denominada de capacidade de sprints repetidos (CSR) (IMPELLIZZERI et. al, 2008), tem sido considerada como um dos principais componentes da aptidão física do jogador de futebol (BISHOP et al., 2004; SPENCER et al., 2005) e futsal (BARBERO-ALVAREZ et al., 2008; CASTAGNA et al., 2009).

No futebol cerca de $1-11 \%$ do total da distância percorrida no jogo é constituído de sprints (BANGSBO, 1991), o que corresponde de a 0,5 - 3\% do tempo efetivo do jogo. Já no futsal, Barbero-Alvarez et al., (2008) demonstraram que os atletas percorrem cerca de $8,9 \%$ do total da distância do jogo em sprints.

A fadiga causa uma redução na capacidade do músculo em gerar força (RAHNAMA et al., 2003) e interfere na rapidez e no controle fino do movimento (JOHNSTON et al., 1998). Na presente pesquisa a CSR foi analisada através do índice de fadiga (IF) e da potência e não foram encontradas diferenças significativas entre as modalidades. Estudos demonstram que 0 IF abaixo de $10 \%$ indica a capacidade dos atletas em manter a performance anaeróbia e, possivelmente, não sofrerem com os efeitos da fadiga. Valores acima de $10 \%$ apontam que 0 atleta necessita melhorar sua tolerância aos esforços anaeróbios intermitentes (ZACHAROGIANNIS et al., 2004).

Um fator que pode explicar a manutenção dos níveis de potência após os sprints é a capacidade de tamponamento da acidez muscular provocada pela presença elevada dos íons $\mathrm{H}+$, considerada um importante atributo para a CSR (BISHOP, 2004). No futsal, a degradação do ATP, na maioria dos casos, é mais acentuada do que a ressíntese de $\mathrm{ADP}+\mathrm{Pi}$, o que pode levar a um quadro de fadiga local acentuada (ARAÚJO et al., 1996) mas, devido a característica da modalidade na qual permite a troca ilimitada de atletas durante a partida, possibilitando um repouso tanto ativo quanto passivo, facilitaria uma melhor ressíntese dos níveis de creatinafosfato. Já no futebol, com sua característica de maior volume e menor intensidade, o atleta consegue fazer a recuperação durante a partida. Essas adaptações podem ser obtidas através do treinamento de elevada intensidade (ROSS et al., 2001), como no caso do futebol e futsal.

\section{Conclusão}

Em conclusão, os atletas de futsal e futebol apresentaram valores similares quanto às variáveis antropométricas de massa corporal, estatura e \% de gordura. Entretanto, as variáveis fisiológicas de $\mathrm{FC}_{\mathrm{LV}} ; \mathrm{VO}_{2 \max } ; \mathrm{VO}_{2 \mathrm{LV}}$ e \%VO $\mathrm{Vmax}_{2}$, apresentaram diferenças significativas entre as modalidades, demonstrando de forma peculiar que, além de uma capacidade anaeróbia mais alta, os atletas de futsal também obtiveram níveis de capacidade aeróbia superiores aos atletas de futebol. Contudo, os atletas de futebol, devido às diferentes posições, tempo e área de jogo, necessitam de uma maior contribuição do metabolismo aeróbio.

Em relação à variável física testada, a capacidade de sprint repetido (CSA), não houve diferença, o que pode ser explicada pela eficiência da capacidade de tamponamento da 
acidez muscular após a realização dos sprints em ambas as modalidades.

Dessa forma, os resultados da presente pesquisa demonstraram que, apesar da similaridade motora entre os esportes, a intensidade de uma partida de futsal é mais acentuada, levando à diferenças significativas entre as características fisiológicas de atletas de futsal e futebol. Essas discrepâncias apresentadas demonstram a necessidade de formação de diferentes métodos de treinamento entre os esportes.

A ausência de estudos comparando variáveis físicas e fisiológicas entre as modalidades é evidente, assim, mais estudos devem ser desenvolvidos com o intuito de trazer novas informações que venham a auxiliar os profissionais de ambas as modalidades.

\section{Referências}

ALEXANDER, M. J. L. The relationship between muscle strength and sprint kinematics in elite sprinters. Canadian Journal of Sport sciences, Downsview, v. 14, n. 3, p. 148-157, 1989.

ARAÚJO, T. L.; ANDRADE, D. R.; FIGUEIRA JÚNIOR, A, J.; FERREIRA, M. Demanda fisiológica durante o jogo de futebol de salão, através da distância percorrida. Revista da APEF de Londrina, Londrina, v. 11, n. 19, p. 12-23, 1996.

ARNASON, A.; SIGURDSSON, S. B.; GUDMUNDSSON, A.; HOLME, I.; ENGEBRETSEN, L.; BAHR, R. Physical fitness, injuries, and team performance in soccer.

Medicine and Science in Sports and Exercise, Madison, v. 36, n. 2, p. 278-285, 2004.

AVELAR, A.; SANTOS, K. M.; CYRINO, E. S.; CARVALHO, F. O.; DIAS, R. M. R.; ALTIMARI, L. R.; GOBBO, L. A. Perfil antropométrico e de desempenho motor de atletas paranaenses de Futsal de elite. Revista Brasileira de Cineantropometria e Desempenho Humano, Florianópolis, v. 10, n. 1, p. 76-80, 2008. Disponível em: <http://bases.bireme.br/cgibin/wxislind.exe/iah/online/?IsisScript=iah/iah.xis\& SrC $=$ google\&base $=$ LILACS\&lang $=$ p\&nextAction $=$ In k\&exprSearch $=490599$ \&indexSearch $=\mid \mathrm{D}$ >.

Acesso em: 02 mar. 2010.

BANGSBO, J.; NORREGAARD, L.; THORSOE, F. Activity profile of competition soccer. Canadian
Journal of Sport sciences, Downsview, v. 16, n. 2, p. 110-116, 1991.

BANGSBO, J. Energy demands in competitive soccer. Journal of Sports Sciences, London, v. 12, p. 5-12, 1994.

BARBERO-ÁLVAREZ, J. C.; GIMENEZ, L.; CORONA, P.; MANONELLES, P. Necesidades cardiovasculares y metabolicas del futbol-sala: analisis de la competicion. Apunts: Educación física y deportes, Barcelona, n. 67, p. 45-53, 2002. Disponível em:

$<$ http://dialnet.unirioja.es/servlet/articulo?codigo $=2$ 42815>. Acesso em: 06 abr. 2010.

BARBERO-ÁLVAREZ, J. C.; SOTO, V. M.; BARBERO-ÁLVAREZ, V.; GRANDA-VERA, J. Match analysis and heart rate of futsal players during competition. Journal of Sports Sciences, London, v. 26, n. 1, p. 63-73, 2008. Disponível em:

$<$ http://dx.doi.org/10.1080/02640410701287289>. Acesso em: 06 abr. 2010.

BARBERO-ÁLVAREZ, J. C.; D'OTTAVIO, S.; GRANDA, V. J.; CASTAGNA, C. Aerobic fitness in futsal players of different. Journal of Strength and Conditioning Research, Champaign, v. 23, n. 7, p. 2163-2166, 2009.

BEAVER, W. L.; WASSERMAN, K.; WHIPP, B. J. On-line computer analysis and breath-by-breath graphical display of exercise function tests.

Journal of Applied Physiology, Washington, v. 34, n. 1, p. 128-132, 1973.

BISHOP, D.; EDGE, J.; GOODMAN, C. Muscle buffer capacity and aerobic fitness are associated with repeated-sprint ability in women. European Journal of Applied Physiology, Berlin, v. 92, n. 4, p. 540-547, 2004. Disponível em: <http://dx.doi.org/10.1007/s00422100411501>. Acesso em: 06 abr. 2010.

BOSCO, C.; TIHANYI, J.; VIRU, A. Relationships between weld fitness test and basal serum testosterone and cortisol levels in soccer players. Clinical Physiology, Oxford, v. 16, p. 317-322, 1996.

CAIOZZO, V. J.; DAVIS, J. A.; ELLIS, J. F.; AZUS, J. L.; VANDAGRIFF, R.; PRIETTO, C. A. A comparison of gas exchange indices used to detect the anaerobic threshold. Journal of Applied Physiology, Washington, v. 53, n. 5, p. 1184-1189, 1982. 
CASAJUS, J. A. Seasonal variation in fitness variables in professional soccer players. Journal of Sports Medicine and Physical Fitness, Turin, v. 41, n. 4, p. 463-469, 2001.

CASTAGNA, C.; D'OTTAVIO, S.; VERA, J. G.; ALVAREZ, J. C. Match demands of professional futsal: a case study. Journal of Science and Medicine in Sport, Belconenn, v. 12, n. 4, p. 490494, 2009. Disponível em:

$<$ http://dx.doi.org/10.1016/i.jsams.2008.02.001>. Acesso em: 06 abr. 2010.

DOWSON, M. N.; NEVILL, M. E.; LAKOMY, H. K.; NEVILL, A. M.; HAZELDINE, R. J. Modelling the relationship between iaokinctic muscle strength and sprint running performance. Journal of Sports Sciences, London, v. 16, n. 3, p. 257-265, 1998. Disponível em: <http://dx.doi.org/10.1080/026404198366786>. Acesso em: 06 abr. 2010.

EKBLOM, B. Applied physiology of soccer. Journal of Sports Sciences, London, v. 3, n. 1, p. 50-60, 1986.

GORDON, C. C.; CHUMLEA, W. C.; ROCHE, A. F. Stature, recumbent length and weight. In: LOHMAN, T. G.; ROCHE, A. F., MARTORELL, R. Anthropometric standardization reference manual. Champaign, Illinois: Human Kinetics, 1988. p. 3-8.

GOROSTIAGA, E. M.; LLODIO, I.; IBÁÑEZ, J.; GRANADOS, C.; NAVARRO, I.; RUESTA, M.; BONNABAU, H.; IZQUIERDO, M. Diferences in physical Wtness among indoor and outdoor elite male soccer players. European Journal of

Applied Physiology, Berlin, v. 106, n. 4, p. 483491, 2009. Disponível em:

<http://dx.doi.org/10.1007/s00421-009-1040-7>. Acesso em: 06 abr. 2010.

HELGERUD, J.; ENGEN, L. C.; WISLOFF, U.; HOFF, J. Aerobic endurance training improves soccer performance. Medicine and Science in Sports and Exercise, Madison, v. 33, n. 11, p. 1925-1931, 2001.

IMPELLIZZERI, F. M.; RAMPININI, E.; CASTAGNA, C.; BISHOP, D.; BRAVO, D. F.; TIBAUDI A. U. Validity of a repeated-sprint test for football. International Journal of Sports

Medicine, Stuttgart, v. 29, n. 11, p. 899-905, 2008.

JACKSON, A. S.; POLLOCK, M. L. Practical assessment of body composition. Physician and
Sports medicine, Minneapolis, v. 13, p. 76-90, 1985.

JOHNSTON, R. B.; HOWARD, M. E.; CAWLEY, P. W.; LOSSE, G. M. Effect of lower extremity muscular fatigue on motor control performance. Medicine and Science in Sports and Exercise, Madison, v. 30, n. 12, p. 1703-1707, 1998.

LEAL JUNIOR, E. C. P.; SOUZA, F. B.; MAGINI, M.; MARTINS, R. A. B. L. Estudo comparativo do consumo de oxigênio e limiar anaeróbio em um teste de esforço progressivo entre atletas profissionais de futebol e futsal. Revista Brasileira de Medicina e Esporte, São Paulo, v. 12, n. 6, p. 323-326, 2006. Disponível em: http://www.scielo.br/scielo.php?script=sci arttext\& pid=S1517-86922006000600005. Acesso em: 15 jan. 2010.

LIMA, A. M. J.; SILVA, D. V. G.; SOUZA, A. O. S. Correlação entre as medidas direta e indireta do $\mathrm{VO}_{2 \max }$ em atletas de futsal Revista Brasileira de Medicina e Esporte, São Paulo, v. 11, n. 3, p. 164-166, 2005. Disponível em: http://www.scielo.br/scielo.php?pid=S151786922005000300002\&script=sci arttext. Acesso em: 03 abr. 2010.

NEWMAN, M. A.; TARPENNING, K. M.; MARINO, $F$. E. Relationships between isokinetic knee strength, single-sprint performance, and repeatedsprint ability in football players. Journal of strength and conditionjng research, Philadelphia, v. 18, n. 4, p. 867-872, 2004.

RAHNAMA, N.; REILLY, T.; LEES, A.; GRAHAMSMITH, P. Muscle fatigue induced by exercise simulating the work rate of competitive soccer. Journal of Sports Sciences, Champaign, v. 21, n. 11, p. 933-942, 2003. Disponível em: $<$ http://dx.doi.org/10.1080/0264041031000140428 >. Acesso em: 06 abr. 2010.

REILLY, T.; BALL, D. The net physiological cost of dribbling a soccer ball. Research Quarterly for Exercise and Sport, Washington, v. 55, n. 3, p. 267-271, 1984. Disponível em:

http://www.eric.ed.gov/ERICWebPortal/search/det ailmini.jsp? nfpb=true\& \&ERICExtSearch Search Value 0=EJ305988\&ERICExtSearch SearchType $0=$ no\&accno=EJ305988. Acesso em: 18 maio 2010.

RIENZI, E, B.; REILLY, T.; CARTER, J. E.; MARTIN, A. Investigation of anthropometric and work-rate profiles of elite South American international soccer players. Journal of Sports Medicine and Physical Fitness, Torino, v. 40, n. 2, p. 162-169, 2000. 
ROSS, A.; LEVERITT, M.; RIEK, S. Neural

influences on sprint running training: adaptations and acute responses. Journal of Sports

Sciences, London, v. 31, n. 6, p. 409-425, 2001.

SIRI, W. E. Body composition from fluid space and density. In: BROZEK, J.; HANSCHEL, A (Eds.). Techniques for measuring body composition. Washingtin: National Academy of Science, 1961.

SPENCER, M.; BISHOP, D.; DAWSON, B.; GOODMAN, C. Physiological and metabolic responses of repeated-sprint activities. Journal of Sports Sciences, London, v. 35, n. 12, p. 10251044, 2005. Disponível em:

http://cat.inist.fr/?aModele=afficheN\&cpsidt=17405 036. Acesso em: 22 fev. 2010.

STOLEN, T.; CHAMARI, K.; CASTAGNA, C.; WISLOFF, U. Physiology of soccer an update. Sports Medicine, Auckland, v. 35, n. 6, p. 501536, 2005.

ZACHAROGIANNIS, E.; PARADISIS, G.;

TZIORTZIS, S. An evaluation of tests of anaerobic power and capacity. Medicine and Science in Sports and Exercise, Madison, v. 36, n. 5, p. 116, 2004.

\section{Endereço:}

Sergio Gregorio da Silva

Rua Coração de Maria, 92 Jd. Botânico

Curitiba PR Brasil

80215-370

Fone: +55 (41) 3360-4331

Fax: +55 (41) 3360-4336

e-mail: sergiogregorio@ufpr.br

Recebido em: 17 de setembro de 2010.

Aceito em: 20 de setembro de 2011. 